François Provenzano*

\title{
Politiques de la sémiotique: Flux et reflux de la critique idéologique chez A. J. Greimas
}

DOI 10.1515/sem-2016-0177

Résumé: L’article part d'un contraste entre deux articles de circonstance signés par Greimas en 1956 pour l'un (« Pour une sociologie du langage »), en 1980 pour l'autre (« Roland Barthes : une biographie à construire »), en montrant la modulation qu'y subissent les topiques d'un substrat marxiste du projet scientifique de la sémiotique : la conciliation entre science et idéologie et de la fonction sociale de l'intellectuel. Ces topiques sont ensuite déclinées au fil de la lecture d'autres travaux du tournant des années 1960-1970, relativement périphériques par rapport au canon de la théorie greimassienne. En témoignant d'un dialogue de la sémiotique avec ses marges disciplinaires (l'histoire, l'ethnologie, la sociolinguistique), ces travaux manifestent un ancrage fort de la réflexion de Greimas dans le terreau d'une critique idéologique historiquement située.

Mots-clés: Marxisme, Roland Barthes, Idéologie, Mai 68, Description sémiotique, Sujet collectif

Abstract: The starting point of the article is a contrast between two topical articles written by Greimas one in 1956 ("Pour une sociologie du langage") and the other in 1980 ("Roland Barthes: une biographie à construire"), demonstrating the variations that the specifically Marxist topics within the scientific study of semiotics are subject to: the reconciliation between science and ideology and the social function of the intellectual. These topics are then dealt with in other works from the 1960s and 1970s, which are associated in some way with the canon of Greimas's theory. Bearing witness to a dialogue of semiotics with its related fields (history, ethnology, sociolinguistics), these works reveal that Greimas's thoughts are strongly rooted in the breeding ground of a historically situated ideological criticism.

Keywords: Marxism, Roland Barthes, Ideology, May 68, Semiotic description, Collective subject

*Corresponding author: François Provenzano, Université de Liège,

E-mail: Francois.Provenzano@ulg.ac.be 


\section{1956-1980 : le flux et le reflux}

En 1956, Greimas publie dans le premier numéro d'Arguments, une revue animée du projet d'une réflexion épistémologique sur les sciences humaines à partir du marxisme et comptant alors également sur la participation de l'ami Roland Barthes, un compte rendu très engagé sur l'ouvrage de Marcel Cohen, Pour une sociologie du langage. Greimas donne ce même titre à son propre papier (Greimas 1956a) et y exprime avec force les attentes qu'il projetait sur un tel titre : en particulier une réconciliation de la linguistique structurale saussurienne et de la linguistique historique, par leur confrontation au marxisme ${ }^{1}$. Cette attente est fortement déçue à la lecture de l'ouvrage de Cohen, marqué, selon Greimas par un " refus de considérer la langue comme une institution sociale » (Greimas 1956a : 17), et une absolutisation des «faits linguistiques », considérés comme distincts des faits sociaux. Or, pour Greimas une « sociologie du langage marxiste » - ce sont ses termes - devrait répondre aux postulats épistémologiques suivants :

[Elle] partirait [...] d'une conception globale du langage et non des faits de langue comme le fait Marcel Cohen ; elle chercherait à décrire les structures des grands ensembles, à dégager les régularités de leur développement, et ne se contenterait pas de leur énumération. Une telle conception, enfin, ne pourra jamais être panchronique, étudiant les langues en dehors du temps et du contexte social concret, mais historique [...]. (Greimas 1956a : 18)

Comme exemple, il cite notamment le cas de la « langue populaire » française, qui a joué le rôle de " principal moteur du développement historique de l'ensemble linguistique » (Greimas 1956a : 19).

La conception globale d'un ensemble linguistique qui ne serait pas le simple instrument transmettant mécaniquement les contenus idéologiques, mais fonctionnant comme une superstructure autonome lui paraît encore étayée par « l'élaboration du concept de l'écriture par R. Barthes »(Greimas 1956a : 19), dont le fameux Degré zéro a paru en 1953, et qui oblige nécessairement à amender, aux yeux de Greimas la doctrine linguistique soviétique de Marr.

Enfin, cette critique nourrie du livre de Cohen se place également sous le signe d'une interrogation liminaire sur les postures d'auteur attendues Greimas se demandant ainsi si Cohen allait afficher « le courage de l'intellectuel marxiste [...] l'inconscience du savant pour qui l'adhésion au Parti n'implique

1 C'est le même vœu qu'il formule, en cette même année 1956, dans son célèbre article du Français moderne, «L’actualité du saussurisme » (Greimas 1956b). 
pas nécessairement la révision de sa science par rapport au marxisme ? ou enfin la fidélité à toute épreuve au maître défunt de la linguistique soviétique ? » (Greimas 1956a : 16).

Cette note de lecture datant des débuts de Greimas n'appartient pas vraiment à ce qu'on peut considérer comme étant le canon de l'œuvre greimassienne. Elle n'a pas fait l'objet d'une republication dans l'un des volumes phares de l'auteur ; par ailleurs, le ton très engagé qui la caractérise est loin des puissantes modélisations qui feront école dès Sémantique structurale, dix ans plus tard. Surtout : les références explicites au marxisme et aux enjeux idéologiques de l'activité scientifique ne laisseront guère de trace sur l'image globale que donnera la sémiotique de la future École de Paris. Or, loin de constituer une sorte de préhistoire de la sémiotique greimassienne, un paradigme différent de celui que retiendra surtout la postérité, les préoccupations qui s'y manifestent nous paraissent, d'une certaine manière, fondatrices du projet théorique de Greimas. Tout du moins, elles lui donnent sa basse continue c'est, en forçant un peu les choses, l'hypothèse générale que nous souhaiterons défendre ici -, au point qu'elles pourront se rendre à nouveau lisibles, malgré leur enfouissement sous un appareil théorique et terminologique perçu comme strictement sémiotique, à la faveur d'un événement aussi radical pour Greimas que le décès de son ami Roland Barthes, en 1980.

Dans le texte d'hommage qu'il publie à cette occasion dans Actes sémiotiques-Bulletin ${ }^{2}$, Greimas fait retour sur l'engagement intellectuel qui, audelà sans doute de l'amitié, le liait à Barthes : " Nous sommes restés [...], pendant une bonne vingtaine d'années, “compagnons de route” partageant un "projet de vie" commun, fondé sur la quête du sens et l'exigence d'une éthique. » (Greimas 1980 : 3) Contre les lectures hédonistes qui ont marqué la réception du "second Barthes », Greimas affirme l'unité morale de l'œuvre de son ami, qui dessine selon lui un « parcours de l'échec » (Greimas 1980 : 3).

On est frappé de voir dans ces lignes le vocabulaire technique de la description sémio-narrative s'appliquer à du vécu, à un matériau biographique et socio-historique très investi, et particulièrement dysphorique:

Exemplaire, cette vie qui s'organise comme un parcours tragique : exemplaire comme une nécessité interne du sujet Roland Barthes ; exemplaire comme l'illustration du désastre d'une génération. / Et, pour couronner le tout, cette dérisoire épreuve glorifiante : le succès à grand tirage et la reconnaissance d'honnêtes gens. (Greimas $1980: 7$ )

2 Lui aussi, comme le précédent, constitue sans doute l'un des éléments de la bibliographie de Greimas dont la portée a pu être perçue comme strictement conjoncturelle - il est pour cela aussi devenu difficilement accessible. Nous remercions ici très chaleureusement Maria Giulia Dondero et Jacques Fontanille de nous avoir permis d'en avoir une copie. 
Comme un double miroir déceptif et cruellement ironique, tendu à la fois sur le substrat historico-biographique (le " parcours tragique » de Barthes et le " désastre d'une génération ») et sur le vocabulaire sémiotique qui en rend compte (« sujet Roland Barthes », « dérisoire épreuve glorifiante »), ces lignes font résonner le projet scientifique de la sémiotique avec le parcours biographique de Barthes (et, à travers lui, de Greimas), marqué par une exigence éthique en même temps que par une profonde désillusion.

Cette désillusion est celle de la « fin de cette sémiologie éthique et, de façon plus générale, d'une certaine manière d'être intellectuel » (Greimas 1980 : 6), que l'auteur associe très précisément au tournant de Mai 68, au «vide idéologique » et à la "mode rétro » qui en sont issus (Greimas 1980 : 4). L'attention accordée au mot intellectuel (les italiques sont de Greimas) nous reporte au compte rendu du 1956, aux espoirs qu'il portait et aux options qu'il dessinait au chercheur en sciences humaines et à sa bonne conscience. La « bonne conscience » sémiologique, c’est celle qui voit la réconciliation de la science et de l'idéologie ; voilà ce qu'a réussi à faire, " pour un temps », Roland Barthes:

La démarche barthésienne qui ne dévoile les contenus "profonds" que pour les condamner se déploie bientôt, lorsqu'elle s'attaque, comme dans ses Mythologies, aux signes sociaux de l'aliénation, comme une vaste entreprise de salubrité publique. Ce projet de démythification du discours social confère en même temps une dignité certaine à la sémiologie : les procédures d'explication, formes appropriées du faire scientifique, se trouvent reconnues, valorisées même, parce que mises au service d'une éthique sociale de lucidité libératrice. L'intellectuel, réconciliant "science" et "idéologie", savoir-faire et faire-savoir, retrouve, pour un temps, sa bonne conscience. (Greimas $1980: 5-6)$

Ici encore, le lexique greimassien de la « profondeur »-associé traditionnellement aux structures narratologiques - et l'attention portée au «faire scientifique » embrayent sur d'autres obsessions de l'auteur : la justification sociale du projet sémiologique et la place de l'intellectuel face aux formes de l'aliénation.

En effet, cette « dignité » que Greimas voit dans la sémiologie barthésienne apparaît bien comme ce qu'il a lui-même poursuivi dans son propre travail, en cherchant cependant à traduire dans un vocabulaire rigoureusement scientifique les termes, chargés d'hypothèques, de la critique marxiste traditionnelle. Par exemple, la notion d'articulation nous semble être le produit d'une telle traduction. Que l'on compare, pour s'en convaincre, les deux extraits suivants ; dans le premier, tiré de l'hommage à Barthes déjà cité, Greimas explicite le projet de l'auteur du Michelet ; dans le second, tiré de "Sémiotique et communications 
sociales »(Greimas 1976a [1970] $\left.{ }^{3}\right)$, Greimas formule pour lui-même le problème d'une sémiotique de la culture de masse:

Renonçant à imposer [...] le déplacement du sens de style, Barthes n'en poursuit pas moins son exploration en tant qu'articulation sémiotique de la masse thymique. (Greimas 1980 : 5 ; nous soulignons)

[...] le problème qui nous préoccupe en premier lieu est de savoir non seulement quelle est l'organisation topologique de l'univers sémantique qui recouvre une communauté culturelle donnée, mais surtout si une organisation particulière de cet univers entraîne, par voie de conséquence, une articulation originale de la "masse sociale", supposée informe pour les besoins de la cause [...]. (Greimas 1976a [1970] : 51 ; nous soulignons)

Certes, à propos du Michelet, il s'agit de la « masse thymique » d'un individu mais un individu justement coupé de son substrat biographique pour être appréhendé par le type d' " articulation sémiotique " qu'il donne à lire dans son œuvre. Dans le second extrait, s'il s'agit bien de se situer à l'échelle d'une communauté culturelle, l'objet visé demeure un " univers sémantique ", considéré là aussi pour l'articulation qu'il propose ${ }^{4}$.

Bref, ces deux premiers exercices de lecture nous engagent sur la voie d'autres textes qui, situés entre ces deux bornes (1956/1980) donneraient une certaine consistance aux quelques pistes dégagées jusqu’à présent.

\section{Hypothèses de lecture}

Ces pistes tiennent, pour l'essentiel et en guise de récapitulation transitoire, aux propositions suivantes, qu'on voudra bien considérer dans toute leur naïveté vague et péremptoire :

- le projet scientifique de la sémiotique greimassienne s'ancre dans le substrat d'un engagement politique marxiste ;

3 Comme l'indique la note qui figure dans l'édition de cet article dans Sémiotique et sciences sociales, le texte " est la refonte complète d'une conférence donnée en octobre 1970 à Milan, dans le cadre du Convegno Nazionale sur "Stato e tendenze attuali della ricerca sulle comunicazioni di massa” et parue dans l'Annuario 1970 de l'Instituto Agostino Gemelli, Milan ». Pour ce texte, comme pour les autres à destin éditorial similaire, nous indiquons la date de l'édition citée et, entre crochets, la date de la première édition - même lorsqu'il y a eu, comme ici, des modifications entre les deux versions.

4 Dans ce même texte, l'auteur affirme comme un "besoin réel " le projet d'une " enquête sémiotique sur les dimensions et les articulations significatives des macro-sociétés actuelles » (Greimas 1976a [1970] : 60). 
- ce substrat fournit une série de topiques, que la discipline sémiotique a traduites en problématiques conceptuelles ;

- cette traduction vise à affronter la question de la conciliation entre science et idéologie et de la fonction sociale de l'intellectuel (et la manière d'assumer cette fonction) ;

- les topiques prises en charge concernent également la distinction entre des structures de surface et des structures profondes et les effets de (dé-) sémantisation (mythologique, idéologique, axiologique) qui en découlent, la constitution des sujets du faire (en particulier des sujets collectifs populaires), le pouvoir de transformation politique associé à ces sujets et la question de l'historicité des structures ;

- l'articulation entre ces différentes topiques, en particulier les rapports entre histoire, idéologie et science, assume un caractère spiralaire : la réflexion sur les rapports entre science et idéologie fait interroger sur le faire scientifique, et cette interrogation oblige elle-même à prendre en compte l'historicité et l'événementialité de ce faire ;

- la positivité de ce projet scientifique, tout comme son reflux déceptif, sont liés aux épisodes de Mai 68 et à la figure de Roland Barthes.

Ces quelques items appelleraient un chapelet de précautions et de nuances. Puisqu'il ne s'agira pas, dans cet article, de venir à bout de cette problématique, mais plutôt d'en esquisser l'éventuelle pertinence, nous nous contenterons de préciser que la démarche à laquelle nous invitons ne consiste pas à « démasquer » ce qui serait le « vrai » Greimas supposément plus « authentique » que celui qu'aurait conservé la doxa greimassienne. L'ambition est plutôt d'éclairer des éléments de la genèse d'un projet scientifique, que l'auteur lui-même ou, plus justement, ses lecteurs, ont (pour de bonnes ou mauvaises raisons, consciemment ou inconsciemment) refoulés de leur horizon d'attente dudit projet. En l'occurrence, il s'agirait de fragments d'un imaginaire politique qui, une fois intégrés dans le réseau terminologique d'interdéfinitions, ont perdu leur charge et leur lisibilité initiales. L'enjeu n'est donc pas tant de faire de Greimas un (crypto-)marxiste, mais de cerner mieux ce que sa théorisation doit à un vocabulaire et à un imaginaire (reconnus comme) autres. Appliquée à la sémiotique (greimassienne), cette démarche trouve une ultérieure justification dans le fait qu'elle apparaît comme une manière de réhistoriciser une théorie.

Dans ce conditionnement, Roland Barthes a assurément joué un rôle de premier plan. On a souvent répété l'influence que Greimas avait exercée sur lui en lui faisant découvrir Saussure à Alexandrie. Il ne serait sans doute pas inutile de considérer également la réciproque inverse, en s'avisant notamment 
des nombreuses références, souvent stratégiques, que Greimas fait au travail de Barthes.

Cela dit, l'éclairage apporté ici sera forcément très partiel. Outre les deux textes évoqués en introduction, nous convoquerons quelques travaux rassemblés dans Sémiotique et sciences sociales. La particularité de ces textes, au-delà du fait qu'ils abordent (plus frontalement qu'ailleurs) les quelques topiques listées ci-dessus, tient également à leurs contextes institutionnels de production : il s'agit d'interventions orales données par Greimas dans des cadres disciplinaires peu liés à la spécialité sémiotique (histoire, ethnologie, communications de masse, sociolinguistique), aux lendemains de Mai 68, hors de France (en Allemagne et en Italie) ; dans la plupart des cas, ces interventions ont fait l'objet d'une première publication en actes de colloque, puis d'une refonte pour la publication dans le recueil Sémiotique et sciences sociales. Ces facteurs de décentrement favorisent selon nous l'affleurement d'une politique de la sémiotique telle que nous l'envisageons ici. C'est bien à ces affleurements que nous nous en tiendrons, en procédant, pour l'essentiel, à une simple lecture des textes, sur le modèle de ce que nous avons proposé en introduction. Les citations seront forcément nombreuses et cet article assumera par moment les allures d'un collage, dont nous croyons cependant aux vertus de remise en perspective ou de rafraîchissement.

Il demeure que l'enquête se devrait d'être considérablement approfondie, dans plusieurs directions. En amont, il faudrait convoquer tout le travail de Thomas Broden sur les « années de formation » de Greimas (Broden 2015 ; voir aussi Broden 2011), pour voir en quoi l'attention aux enjeux de la collectivité s'ancre dans un intérêt pour le folklore populaire (lituanien). En aval, il faudrait ajouter à l'hommage à Barthes (Greimas 1980) tous les autres témoignages plus ou moins rétrospectifs donnés par Greimas sur son parcours, ses engagements, et les liens entre son projet théorique et ses propres orientations idéologiques (voir notamment Chevalier et Encrevé 2006). Enfin, ces témoignages seraient utilement complétés par une enquête de réception approfondie, qui envisagerait le point de vue d'autres acteurs du temps (voir notamment Arrivé 1993).

De tout cela hélas, le présent article ne peut faire état ; gageons qu'il puisse cependant, malgré son aspect fragmentaire et sans doute parfois impressionniste, montrer l'intérêt d'une histoire sociale de la sémiotique, en prise directe avec son actualité épistémologique. En effet, on se sera avisé que les inquiétudes de Greimas quant à la "dignité » de sa discipline et à sa portée éthique ne manquent pas de présenter quelques résonances plus ou moins explicites avec les voies explorées par certains travaux récents. 


\section{Structures et description}

Alors même qu'on voit en Greimas celui par qui la sémiotique s'est institutionnalisée en France comme discipline autonome et identifiable comme telle dans le champ des humanités, il est frappant de constater que le contexte du début des années 1970 le voit prendre part, à l'étranger, à une série de collectifs profondément marqués par ce qu'il conviendrait aujourd'hui d'appeler une ouverture transdisciplinaire. C'est assurément le cas pour le volume Geschichte-Ereignis und Erzählung, où paraît initialement le texte « Sur l'histoire événementielle et l'histoire fondamentale » (Greimas 1976b [1973]).

Greimas s'y interroge à la fois sur le sens produit par les structures historiques elles-mêmes et sur le fonctionnement du discours historique qui en rend compte. C'est l'occasion pour lui de faire le procès d'une historiographie qui n'aurait pas pris la mesure de "l'héritage de Marx » et de sa description des structures sociales :

Il est à la fois curieux et regrettable de constater que, malgré l'âge déjà respectable de la théorie marxiste de la stratification sociale, l'histoire continue à être écrite, dans ses grandes lignes, de la même manière que par le passé, qu'aucun effort sérieux n’a été fait pour élaborer les descriptions, homogènes et comparables, des différents niveaux structurels des sociétés. / Et pourtant l'héritage de Marx comporte la description, en quelque sorte exemplaire, de la structure économique appelée capitalisme dont le mérite, indépendamment de sa valeur intrinsèque, est d'être un modèle construit et, de plus, un modèle achronique. (Greimas 1976b [1973] : 165-166)

On voit clairement ici que le marxisme fournit le modèle 5 épistémologique d'une " description homogène » de " niveaux structurels », dont les historiens ont jusqu'à présent échoué à considérer la dynamique et les divers degrés de profondeur. Par ailleurs, le postulat de l'achronie est bien associé au « modèle construit ", et non à l'objet d'étude lui-même : il ne s'agit nullement d'arracher cet objet à son historicité, mais au contraire de rendre compte de cette historicité par une procédure construite dont la validité échappe, elle, à la contingence historique.

Ce modèle épistémologique se nourrit également d'un autre apport fondamental, moins enfoui sans doute, mais souvent perçu à partir du seul point de

5 On notera en passant la présence dans cet extrait du même terme exemplaire ( « l'héritage de Marx comporte la description, en quelque sorte exemplaire ») que Greimas attribue avec emphase au parcours de Barthes, dans l'extrait déjà cité (« Exemplaire, cette vie qui s'organise comme un parcours tragique : exemplaire comme une nécessité interne du sujet Roland Barthes ; exemplaire comme l'illustration du désastre d'une génération », [Greimas 1980 : 7]). 
vue de la sémiotique narrative qui s'est construite à partir de lui : l'apport des recherches mythologiques de Dumézil.

\section{Les avatars de l'idéologie}

On pourrait dire que Greimas a trouvé chez Georges Dumézil ce qu'il n'a pas trouvé chez Cohen - l'article qui, dans L'Homme de 1963, rend compte de sa découverte est republié dans $\mathrm{Du}$ Sens en 1970 et est dès lors bien connu. Mais c'est aussi dans un article sur "La littérature ethnique » (Greimas 1976c), initialement conçu comme les conclusions d'un symposium d'ethnologues réunis à Palerme en 1970 et repris ensuite dans Sémiotique et sciences sociales, que Greimas revient sur le substrat mythologique de sa sémiotique, en y explicitant davantage le processus de traduction auquel il a donné lieu'.

La description des mythes ou des contes n'est que le dévoilement du niveau idéologique caché sous les apparences d'un faire anthropomorphe. L'œuvre qui a mis au jour l'idéologie tripartite des sociétés indo-européennes constitue ainsi un des fondements de la sémiotique narrative. [...] J'ai essayé, par la suite, d'opposer les axiologies aux idéologies, de la même manière que les taxinomies s'opposent aux structures narratives : l'idéologie tripartite serait, dans ce cas, considérée comme un modèle axiologique, ce qui n'est qu'un changement terminologique. Cependant, on voit bien que l'utilisation idéologique - et non classificatoire des trois fonctions est également possible. Il s'agirait d'examiner sous cet angle le problème de l'articulation fondamentale des contenus, tels que nous le rencontrons dans l'analyse des modalités du récit : les modalités du pouvoir, du savoir et du vouloir, d'un côté, et les objets de valeur transmissibles, de l'autre. [...] Je voudrais, dans cette perspective, considérer comme non pertinentes certaines interventions ayant cherché à établir la comparaison entre le sens du récit et ses fondements culturels ou idéologiques qui, sous la forme d'un référent, se trouveraient ailleurs. Pour moi, les récits mythiques portent en eux-mêmes leur idéologie. (Greimas 1976c : 203-204)

6 Selon une vue un peu schématique, on pourrait considérer que Sémiotique et sciences sociales donne à lire une sorte d'envers génétique du projet sémiotique, envisagé à partir de ses sources théoriques plurielles, là où $\mathrm{Du}$ Sens, et plus tard $\mathrm{Du}$ Sens II, n'en livrent que la formulation positive, déjà systématisée, disciplinarisée en somme. On pourrait également considérer qu'il y a encore, sous Sémiotique et sciences sociales, une couche rédactionnelle plus « élémentaire », si l'on veut, qui serait celle des versions données par Greimas aux différents collectifs auxquels il a pris part. Un travail philologique de comparaisons entre ces versions laisserait sans doute apparaître de profondes reformulations, et des coupes substantielles. C'est le cas, à toute évidence, de l'article« Sur l'histoire événementielle et l'histoire fondamentale », dont la version originale dans le collectif allemand laisse apparaître un développement plus conséquent des exemples puisés à l'actualité de Mai 68, une insistance sur les apports du substrat marxiste, et des considérations sur les rapports entre science et idéologie (voir infra). 
On retrouve dans cette citation l'étagement en " niveaux ", qui se double ici d'une distinction entre l'apparent et le « caché ». La description se donne ainsi comme un "dévoilement » et la sémiotique narrative s'inscrit ainsi elle-même dans le paradigme du « dévoilement ». Reste que, dans ce paradigme, elle opère une série de déplacements terminologiques et pointe des enjeux nouveaux, parmi lesquels celui du lieu de l'idéologie : l'option ici exprimée par Greimas est de situer ce lieu dans le plan d'immanence du récit, et non d'un hypothétique plan des référents (on notera la prudence de l'expression « sous la forme d'un référent »). Mais cette option n'a rien d'exclusif ni de définitif : c'est l'indigence actuelle des recherches sociologiques qui convainc Greimas de considérer avec guillemets (et avec regrets - « malheureusement », « j’ai bien peur ») le «plan de la "réalité" »:

Évidemment, la question de la comparaison des idéologies avec les domaines isotopes $\mathrm{du}$ plan de la " réalité » pourrait être posée, mais une telle comparaison comporterait comme préalable la description du plan de la « réalité », dont nous ne disposons malheureusement pas et que la sociologie, j'ai bien peur, n'est pas prête à nous fournir. / Cela ne veut pas dire, bien au contraire, que la comparaison entre idéologies, surtout si elles sont décrites de manière isomorphe, ne soit possible. (Greimas 1976c : 204)

C'est donc comme par défaut que la sémiotique narrative peut donner à ses descriptions l'horizon d'un comparatisme idéologico-culturel qui, pour Greimas a toutes les raisons de s'appliquer également « à l'étude des mythologies des sociétés industrielles »(Greimas 1976c : 204). La sémiotique narrative apparaît ainsi comme ce qui permet d'unifier les recherches mythologiques d'un Dumézil à la critique idéologique d'un Roland Barthes (dont les Mythologies sont fréquemment évoquées en exemple par Greimas), à défaut d'une véritable sociologie structurale, et en assumant le postulat d'une immanence narrative de l’idéologie.

Ce postulat est-il suffisant pour donner sa "bonne conscience » au sémioticien et une "dignité » à sa discipline ? Il semble bien que non, lorsqu'on observe la manière dont le projet décrit ci-dessus se formule, dans les mêmes années (1968-1970), sous des étiquettes disciplinaires légèrement décalées par rapport au cadre de la " sémiotique narrative ». Après avoir dit la déception de ses vœux "Pour une sociologie du langage », Greimas adopte lui-même la formule du titre (ou du sous-titre) engagé, en plaidant tantôt « [p]our une sociologie du sens commun » (Greimas 1970 [1968]), tantôt « pour une grammaire socio-sémiotique » (Greimas 1976d [1969]).

Dans les deux textes, la notion de connotation occupe une place centrale, et apparaît comme une autre traduction de l'idéologie dans le cadre d'un projet 
scientifique (après le cas d'axiologie, vu plus haut). Dans les deux textes également, le sous-texte à peine voilé est encore Barthes, cette fois celui de la " Rhétorique de l'image », paru quelques années plus tôt (Barthes 1964). Tandis que Barthes érigeait le terme idéologie en plan d'analyse à part entière de la description scientifique - celui du « domaine commun des signifiés de connotation » (Barthes 1964 : 49) -, Greimas réserve cette tâche à la " socio-linguistique », qui serait « l'étude des langages de connotation sociale »(Greimas 1976d [1969] : 62). Chez Barthes, la mise au jour de signifiés de connotation idéologique avait quelque chose d'intuitif et d'impressionniste. Dans l'hommage déjà cité, Greimas fait en quelque sorte le procès d'une sémiologie « devenue, pour une large part, la description des connotations sociales » :

\footnotetext{
Or, contrairement à ce qui se passe lors de la construction des sémiotiques particulières les connotations ne peuvent être saisies à partir de leurs signifiants, elles demandent à être postulées d'abord comme des signifiés : la part de l'intuition, de la perspicacité du chercheur, il faut le reconnaître, y est alors dominante. (Greimas $1980: 6$ )
}

C'est sans doute pour éviter ce déport intuitionniste et anti-scientifique de la sémiologie que Greimas formule le vœu d'une telle socio-linguistique, dont la « praxis scientifique » est là pour conjurer l'arbitraire de l'interprète et, derrière lui, du « signe socio-linguistique » lui-même:

C'est l'arbitraire du « signe socio-linguistique » qui nous oblige à élaborer, pendant que la description se poursuit, des hypothèses de travail de caractère général qui prennent la forme de modèles socio-linguistiques [...]. La praxis scientifique seule peut effectuer leur validation. (Greimas 1976d [1969] : 76)

De la même manière que la socio-linguistique - ou plus globalement la sociosémiotique et son ambition grammaticale - est là pour ancrer la description des signifiés idéologiques dans des procédures de validation scientifique qui prennent au sérieux la nature sémiotique (c'est-à-dire duelle mais arbitraire) des objets étudiés, ce que Barthes réservait au domaine de la rhétorique, à savoir la « face signifiante de l'idéologie » (Barthes 1964 : 49), est symétriquement intégré dans un cadre global, celui d'une "sémiotique des systèmes connotatifs » qui ne se distinguerait plus d'une « sociologie du sens commun ». Greimas voit en effet dans « ces disciplines qui sont en train de se reconstituer, comme la poétique ou la rhétorique » (Greimas 1970 [1968] : 98) des études spécialisées sur les formes littéraires, dont la problématique « doit être distinguée de l'attitude qu'une société adopte vis-à-vis des signes de son langage » (Greimas 1970 [1968] : 98). Ici encore, on voit bien que le texte littéraire est loin d'être l'horizon disciplinaire que se donne la sémiotique, dont le projet entend toucher au vécu des groupes sociaux. 
En effet, la « reconnaissance du phénomène de la connotation » « permet d'intégrer dans la recherche sémiotique, et de faire bénéficier de la méthodologie de cette dernière, un champ de significations dont l'appréhension scientifique paraît encore impossible et qu'on invoque souvent comme le niveau du vécu et du senti, du quotidien et de l'humain » (Greimas 1970 [1968] : 99-100). La sémiotique fournirait ainsi le socle scientifique pour la description des phénomènes que le lexique marxiste (aliénation et démythification) maintenait en prise avec des jugements de valeur ; ou mieux : la sémiotique tire sa justification scientifique de l'existence d'un « univers culturel de sens commun, connoté dans son ensemble comme la réalité sociale vécue », qui demande à être décrit comme « la manifestation de la structure connotative d'une langue » (Greimas 1970 [1968] : 102).

\section{Désémantisation et resémantisation}

En cherchant à redéfinir sémiotiquement les termes de la problématique idéologique marxiste, tout en maintenant le dédoublement fondamental entre structures profondes et structures de surface, Greimas ouvre la voie, on l'a vu, à une typologie des cultures. C'est, en quelque sorte, outre la technicité descriptive, l'horizon typologique qui assoit la scientificité du projet et lui évite de se ramener à un simple « procès de démythification » (Greimas 1970 [1968] : 102). Il n’empêche que, là encore, la « bonne conscience » du sémioticien ne semble pas entièrement se satisfaire de cet horizon typologique et réintroduise du processuel là où sa science l'avait conduit à voir du systématique, du diachronique là où son modèle le poussait à l'achronie, en somme embraye sa propre praxis sur les dynamiques de sémantisation à l'œuvre dans son objet.

Après l'histoire, la sociologie et la socio-linguistique, c'est l'ethnologie qui sert cette fois de terrain à la réflexion disciplinaire du sémioticien. Dans une conférence au Congrès international d'ethnologie européenne donnée en 1971 et publiée pour la première fois en 1973 (Greimas 1976e [1973]), il développe une réflexion à propos d'objets dits " ethno-sémiotiques ", comme la poésie sacrée ou le folklore populaire, et émet l'hypothèse d'un " parcours typologique », c'est-à-dire d'une projection de la typologie sur la diachronie, qui permet de rejouer l'opposition entre la profondeur et la surface sur le mode d'un procès de « désémantisation ":

Aussi peut-on se demander si un parcours typologique [...] ne mène pas de la poésie sacrée, de nature ethno-sémiotique, à la poésie folklorique, désémantisée d'une certaine manière, et jusqu'à la réapparition de la poésie dite moderne, individualisée et souvent hermétique. 


\begin{abstract}
Nos langues modernes ne possèdent pas de mot pour désigner ces objets mythiques complexes que sont les " chants dansés » ou les "danses chantées » : il n'en est pas de même des sociétés dites archaïques. Aurait-on tort cependant de voir dans certaines formes de la gestualité folklorique (chansons de travail, jeux dansés et chantés, certaines danses populaires) des formes dégradées, désémantisées de la gestualité rituelle d'autrefois ? En adoptant l'hypothèse de la désémantisation qui caractériserait le phénomène folklorique [...], on pourrait [...] chercher à élaborer, à partir des stéréotypes culturels rencontrés au niveau folklorique, des procédures permettant la réactivation de la signification. (Greimas 1976e [1973] : 181)
\end{abstract}

Plusieurs faits remarquables se manifestent dans cet extrait. Il faut souligner d'abord une rhétorique de l'hypothèse prudente, dont témoignent les questions rhétoriques et les approximations expressives, en particulier autour de la notion de désémantisation (« désémantisée d'une certaine manière », « des formes dégradées, désémantisées »). Cette rhétorique laisse entendre que l'auteur s'avance ici sur un terrain dont il ne semble pas encore sûr de vouloir assumer toutes les dimensions. Cette prudence dissimule (mal) à nos yeux deux coups de force, ou plutôt deux sauts épistémologiques majeurs : d'une part, celui d'une linéarisation temporelle des objets ethno-sémiotiques (répartis entre un " autrefois » et un " moderne " auquel s'inclut le « nous » - «nos langues modernes »); d'autre part, celui d'une axiologisation de ce parcours, polarisé selon un processus de désémantisation qui s'apparente à une dégradation - dont témoigne également la mention "souvent hermétique " attribuée à la " poésie dite moderne ". Ce double saut épistémologique a forcément des répercussions sur les enjeux mêmes de la praxis scientifique (ethno-)sémiotique, qui semble inscrite contre la pente désémantisante propre à la modernité, puisque ses procédures devraient permettre la « réactivation de la signification ».

La nature de cette réactivation reste bien sûr ambiguë : assume-t-elle un simple caractère archéologique, ou vise-t-elle à agir dans la profondeur même de la synchronie "moderne " pour la réactiver sémantiquement ? La question n'appelle pas forcément de réponse exclusive, mais gagne sans doute à être posée en écho avec une dernière topique située elle aussi à l'interface entre science et idéologie : celle de la conception du sujet collectif de l'histoire.

\title{
6 Les sujets collectifs de l'histoire
}

On peut remarquer en effet que le pôle " désémantisé » est aussi présenté comme " individualis[é] », là où l'accès plein et entier au sens se fait par le biais de pratiques collectives (ritualisées et même, dirions-nous, davantage incorporées que verbalisées). Notons en passant que les exemples évoqués parmi les « formes 
de la gestualité folklorique (chansons de travail, jeux dansés et chantés, certaines danses populaires) » font immanquablement écho avec les conceptions évoquées par Valentin Vološinov dans le célèbre Marxisme et philosophie du langage, dont la traduction française, aux Éditions de Minuit, alors signée par Mikhail Bakhtine et préfacée par Roman Jakobson, paraîtra l'année suivante, en 1977 : l'idée selon laquelle, pour Vološinov, « l'individu “abstrait” n'a aucune existence en dehors du ou d'un "groupe social” " (Sériot 2011 : 91), est illustrée, dit Vološinov, par certains types " dans les veillées de village, les fêtes populaires en ville, le bavardage des ouvriers pendant la pause à l'heure du déjeuner, etc. » (Vološinov 2010 : 99 ; cité dans Sériot 2011 : 91). C’est le même imaginaire politique $\mathrm{du}$ « populaire » qui imprègne la réflexion d’Émile Benveniste dans son célèbre article sur « L'appareil formel de l'énonciation » (paru originellement en 1970, soit au cœur de la période d'activité considérée ici pour Greimas), lorsqu'il rapporte ainsi les propos de Malinowski à propos de la communion phatique $^{7}$. Faut-il dès lors s'étonner de voir apparaître sous la plume de Greimas le terme benvenistien d'appropriation (sous sa forme verbale « se l'approprie ») pour qualifier ces formes d'incorporation collective de la sémiose ?:

Une dimension complémentaire de la signification, la dimension proxémique, apparaît ainsi : que ce soit dans les chants lituaniens d'attente du printemps où l'appel poétique est renforcé par le lancement itératif, aussi haut que possible, de la chanteuse juchée sur la balançoire, ou dans la farandole provençale [...], où la chaîne humaine explore, dans un mouvement rythmique ininterrompu, la totalité de l'espace communautaire et se l'approprie mythiquement, la gestualité rituelle se présente comme la relation de l'homme au monde. (Greimas 1976e : 182 ; nous soulignons)

Nous connaissons le passage célèbre de l'article « L'appareil formel de l'énonciation », où l'auteur définit cette notion " comme un procès d'appropriation » (Benveniste 1974a [1970] : 82). L'intérêt est de voir que ce terme est précisément celui dont le signifiant insiste dans l'entretien donné par Émile Benveniste aux Lettres françaises, où le linguiste souligne avec emphase l'importance de l'enjeu:

7 « Il vaut la peine de citer quelques passages de cette analyse : [...] Quand les gens s'assoient ensemble auprès d'un feu de village après avoir achevé leur tâche quotidienne ou quand ils causent pour se délasser du travail, ou quand ils accompagnent un travail simplement manuel d'un bavardage sans rapport avec ce qu'ils font, il est clair qu'ici nous avons affaire à une autre manière d'employer la langue, avec un autre type de fonction du discours. [...] la situation en tous ces cas est créée par l'échange de mots [...]. » (Benveniste 1974a : 87). Sur l'analyse de cet imaginaire politique voir Provenzano (2014). 


\begin{abstract}
L'appropriation du langage à l'homme, c'est l'appropriation du langage à l'ensemble des données qu'il est censé traduire, l'appropriation de la langue à toutes les conquêtes intellectuelles que le maniement de la langue permet. C'est là quelque chose de fondamental : le processus dynamique de la langue, qui permet d'inventer de nouveaux concepts et par conséquent de refaire la langue, sur elle-même en quelque sorte. Eh bien ! tout cela c'est le domaine du « sens ». (Benveniste 1974b [1968] : 21)
\end{abstract}

Loin de s'indexer sur une position strictement individualiste - ce que peut laisser penser la définition de « L'appareil formel de l'énonciation », qui insiste sur « le locuteur »-, le propos de Benveniste donne à ce terme appropriation une résonance résolument collective : c'est l'Homme dans sa généralité qui est invité à s’approprier une langue qui lui donnera la capacité créatrice de s'inventer dans la société (Provenzano 2014).

Ce maillage intertextuel est là pour étayer une interprétation du texte de Greimas comme un élargissement, du linguistique au sémiotique (proxémique), du procès d'appropriation collective par lequel un sujet s'humanise, c'est-à-dire se rapporte à autrui et au monde. C'est, chez Greimas ce procès qui distingue les sociétés " ethno-sémiotiques » des sociétés dites tantôt " industrielles », tantôt " modernes », " macro-sociétés », " développées », " socio-sémiotiques », dans une grande labilité terminologique témoignant de l'opération de traduction déjà mentionnée. Les premières donnent aux objets sémiotiques le statut de «productions collectives de sens ", tandis que dans les secondes ils « deviennent essentiellement des objets de consommation individuelle » (Greimas 1976e [1973] : 179).

Or, Greimas précise cette distinction en s'interrogeant sur les formes institutionnelles concrètes que prennent ces pratiques d'appropriation intégratrice qui, dit-il, permettent " l'instauration du groupe social en tant que sujet collectif " (Greimas 1976e [1973] : 185). L'explicitation de l'efficace sociale de tels dispositifs le conduit à évoquer le cas particulier de« la société française » comme macrosociété:

[...] on voit que les sociétés à communication ethno-sémiotique possèdent une forte cohésion sociale. Rien d'étonnant alors que les macro-sociétés, telle la société française, par exemple, n'aient conservé le chant en groupe que dans deux cas précis : à l'école maternelle, lieu du premier apprentissage de la vie sociale, et très partiellement à l'armée, lieu de la soumission de l'individu aux normes et aux finalités sociales. (Greimas 1976e [1973] : 185)

Il ne nous paraît pas anodin que la pointe d'ironie qui perce dans la mention de (fausse) évidence« [r]ien d'étonnant alors » porte implicitement sur le fait que le peu de cohésion sociale qu'il resterait à la société française soit à chercher dans 
ces lieux hyper-institutionnalisés d'aliénation par excellence que sont l'école et l'armée, cibles privilégiées des slogans de Mai 68.

L’interprétation est peut-être ici un peu forcée ; elle entend seulement suggérer que Greimas réfléchit à ces phénomènes d'instauration de sujets collectifs sans les limiter aux pratiques des sociétés « archaïques », objets d'une ethno-sémiotique ou éventuellement d'une approche archéologique du folklore populaire, mais en en cherchant les avatars dans sa réalité socio-historique la plus immédiate : les procédures éprouvées de la sémiotique narrative, mettant notamment en lumière « l'assomption commune des modalités constitutives de la compétence du sujet », sont appliquées ailleurs aux exemples pleinement et fraîchement historiques de « la production des voitures chez Renault » et de « la foule qui manifeste dans les rues de Paris $^{8}$ » (exemple lui-même particularisé ensuite par l'événement de la « Prise de la Bastille ») (Greimas 1976b [1973] : 171-172).

On voit ici clairement comment la modélisation théorique et la sophistication terminologique font retour sur les occurrences historiques pour en décrire le fonctionnement sémiotique, et pour voir surtout l'efficace sociale de ce fonctionnement, en l'occurrence l'émergence d'un sujet collectif comme actant historique dans des contextes de profonde transformation politique.

\section{Pour conclure : transformation, narration et idéologie}

Greimas lui-même, dans les exemples évoqués ci-dessus, ne parle pas de « contextes de profonde transformation politique »; il se contente d'évoquer prudemment, et plus ou moins explicitement, quelques fragments d'histoire que le lecteur ou l'auditeur pourra connoter à sa guise. Il semble bien que cette absence de prise de position explicite de la description sémiotique par rapport aux procès de transformation historique ait été méditée par Greimas ou en tout cas ait fait l'objet d'une forme de repentir : lorsqu'on compare la version de l'article « Sur l'histoire événementielle et l'histoire fondamentale » publiée en

8 L'évocation est un peu plus précise dans la version du texte parue en 1973 dans les actes du colloque Geschichte und Geschichten : « la rue n'est qu'une métaphore qui désigne la foule, un groupe d'individus qui manifestent, qui, parfois, s'attaquent aux policiers, etc. » (Greimas 1973 : 149). De même, le rapport entre ce « sujet collectif» et les individus concrets auxquels il renvoie est évoqué plus précisément : "Dans quelles conditions peut-on dire que telle foule, par exemple, est le sujet hyponymique de la classe ouvrière et telle autre, de la classe estudiantine? » (Greimas $1973:$ 149). 
1976 dans Sémiotiques et sciences sociales à celle datée de 1973 et parue dans le collectif allemand Geschichte-Ereignis und Erzählung, on s'avise en effet que toute la partie finale a été supprimée. Or, c'est dans cette partie finale que l'auteur développe une réflexion sur la transformation en histoire, et surtout sur la manière d'en rendre compte:

[...] assez paradoxalement, le faire historique apparaît comme reproductif, et non comme transformatif, et l'histoire elle-même, avant d'être conçue comme un changement, doit d'abord être comprise comme permanence. [...] Si tant est que les changements historiques peuvent se produire par l'effet de glissements et de distorsions imperceptibles, tout se passe comme si l'homme, pour en rendre compte sur le plan de la signification, ne devait retenir que les pôles extrêmes et imaginer une narration polémique, un nouveau programme, mais idéologique cette fois-ci, dont le sens ultime consiste à rendre compte de la transformation effectuée. (Greimas 1973 : 151-152)

Ces lignes nous donnent à lire un Greimas pris entre science de l'idéologie et idéologie de la science; elles éclairent en quelque sorte la face cachée du projet sémiotique, et comme l'aveu de son impossibilité : l'analyse (et donc, quelque part, la justification) scientifique de la transformation du sens historique, en tant qu'elle échapperait aux effets d'une narration idéologique.

On notera cependant que le sujet de cette narration idéologique n'est plus désigné comme " chercheur », ni comme « intellectuel » ou comme « savant », mais comme «l'homme ». Cette désignation générique nous semble redonner littéralement un sens, anthropologique plus que scientifique, à l'usage des formes narratives:

Il s'agit là de deux types d'interrelations [entre histoire profonde et histoire événementielle] que l'on retrouve tout aussi bien dans les différentes philosophies de l'histoire que dans les formes narratives par lesquelles s'explicite, sur le mode de l'imaginaire, la réflexion de l'humanité sur son propre destin. (Greimas $1973: 153)^{9}$

Autrement dit, en assumant leur fonction de compte rendu idéologique des transformations historiques, ces formes narratives participent au procès de « resémantisation » qui permet à l'homme de s'imaginer comme acteur du monde.

\section{Bibliographie}

Arrivé, M. 1993. Souvenirs scientifiques et autres sur A. J. Greimas. Nouveaux Actes

Sémiotiques. 13-23.

9 Il s'agit également d'un passage absent de l'édition de 1976. 
Barthes, R. 1964. Rhétorique de l'image. Communications 4. 40-51.

Benveniste, É. 1974a. Problèmes de linguistique générale, t. II, Paris, Gallimard, « Tel ». Benveniste, É. 1974b [1968]. Structuralisme et linguistique [Entretien avec Pierre Daix. In Problèmes de linguistique générale, t. 2, 11-28. Paris, Gallimard, « Tel ».

Broden, Th. F. 2011. Toward a biography of Algirdas Julius Greimas (1917-1992). Lituanus, 57(4). Broden, Th. F. 2015. A. J. Greimas : les années de formation. Texto! Textes \& Cultures, 20(2). Chevalier, J.-Cl. \& P. Encrevé. 2006. Combats pour la linguistique, de Martinet à Kristeva. Essai de dramaturgie épistémologique. Paris: ENS.

Greimas, A. J. 1956a. Pour une sociologie du langage. Arguments, 1. 16-19.

Greimas, A. J. 1956b. L'actualité du saussurisme. Le Français moderne, 25. 191-203.

Greimas, A. J. 1970 [1968]. Pour une sociologie du sens commun. In : Du sens, 93-102. Paris: seuil.

Greimas, A. J. 1973. Sur l'histoire événementielle et l'histoire fondamentale. In : Koselleck, R. et W.-D. Stempel (dir.), Geschichte-Ereignis und Erzählung, Munich, Wilhelm Fink, 139-153.

Greimas, A. J. 1976a [1970]. Sémiotique et communications sociales. In : Sémiotique et sciences sociales, 45-60. Paris: Seuil.

Greimas, A. J. 1976b [1973]. Sur l'histoire événementielle et l'histoire fondamentale. In Sémiotique et sciences sociales, 161-174. Paris: Seuil.

Greimas, A. J. 1976c. La littérature ethnique. In Sémiotique et sciences sociales, 189-216. Paris: Seuil.

Greimas, A. J. 1976d [1969]. Des modèles théoriques en socio-linguistique (pour une grammaire socio-sémiotique). In Sémiotique et sciences sociales, 61-76. Paris: Seuil.

Greimas, A. J. 1976e [1973]. Réflexion sur les objets ethno-sémiotiques. In Sémiotique et sciences sociales, 175-185. Paris: Seuil.

Greimas, A. J. 1980. Roland Barthes : une biographie à construire. Actes sémiotiques, Bulletin 13. 3-7.

Provenzano, Fr. 2014. L'imaginaire politique de la théorie de l'énonciation. Langage et société 147. 133-150.

Sériot, P. 2011. Vološinov, la philosophie du langage et le marxisme. Langages 182. 83-97.

Vološinov, V. 2010. Marxisme et philosophie du langage, Patrick Sériot \& Inna TylkowskiAgeeva (eds. \& trans.). Limoges: Lambert Lucas. 